Original Research Paper

\title{
Investigation of Periodic Points in Algebraic Varieties
}

\author{
Ahmed M. Ibrahim and Elhadi E. E. Dalam \\ Department of Mathematics, College of Arts and Science, Al Baha University, Al Mandag, Saudi Arabia
}

\author{
Article history \\ Received: 02-11-2020 \\ Revised: 15-01-2021 \\ Accepted: 18-01-2021 \\ Corresponding Author: \\ Ahmed M. Ibrahim \\ Department of Mathematics, \\ College of Arts and Science, $\mathrm{Al}$ \\ Baha University, Al Mandag, \\ Saudi Arabia \\ Email: aaadam@bu.edu.sa
}

\begin{abstract}
The aims of this study, the researchers inspected that if $X$ an algebraic variety over a field $K$ and $f$ is a $f^{1+2 \varepsilon}(P)=P$ for other $\varepsilon>-1 / 2$, then the aset of periods time is limited and the little one is $1+2 \varepsilon$. Also the researchers confirmed that if $\mathcal{O}$ be a separate estimate ring of characteristics zero with residue field $k$ of characteristics $(2+\varepsilon)$ of integers in $K$ and $(A, m)$ be a local sub- $\mathcal{O}$-algebra $\mathcal{O}^{(2+\varepsilon)^{(1+2 \varepsilon)}}$ of rank $(2+\varepsilon)^{(1+2 \varepsilon)}$, then $(1+2 \varepsilon)=v^{2}(2+\varepsilon)$, $\forall \varepsilon>0$, where $v^{2}$ is the normalized valuation on $K$.
\end{abstract}

Keywords: Algebraic Variety, Discrete Valuation Ring, Boundedness

\section{Introduction}

In this study, the researchers follow the same literature and methods by (Fakhruddin, 2001) with a small change.

Let $X$ be an algebraic difference over a field $K$ and let $f: X \rightarrow X$ be a morphism. A point $P$ in $X(K)$ is $f$-periodic and the smallest such point is called the period of $P$.

\section{Conjecture 1.1}

Let $X$ be a suitable algebraic difference over a finitely generated field $K$ of characteristic zero and $f: X \rightarrow X$ a morphism. Assuming there exists a subset $S$ of $X(K)$ which is Zariski dense in $X$ and such that $f$ induces a bijection of $S$ onto itself. Then $f$ is an automorphism.

This can be easily confirmed for $X=P^{1+2 \varepsilon}$ or $X$ an abelian variety using heights and the Mordell-Weil theorem respectively.

\section{Theorem 1.2}

Let $X$ be a proper difference over a field $K$ which is finitely generated over the prime field and let $f: X \rightarrow X$ be a morphism:

(i) If characteristic of $K$ is equal zero then the set of periods of all $f$-periodic points in $X(K)$ is finite

(ii) If characteristic of $K$ is equal $2+\varepsilon \neq 0$ then the prime to $p$ parts of the set of periods is finite i.e., there exists $\varepsilon>-\frac{1}{2}$ such that all the $f^{1+2 \varepsilon}$-periodic points in $X(K)$ have periods which are powers of $2+\varepsilon$

Many special conditions of that result have long been reported, the first theorem setup by (Northcott, 1950) proving the finiteness of the number of periodic points in specific conditions. For more details of periodic points check (Morton and Silverman, 1994; Batoréo, 2018; Pravec, 2019).

\section{Remark 1.3}

The researchers do not know whether the periods can really be unbounded if characteristic of $K>0$.

The theorem is clear if $K$ is a finite field and the researchers reduce the general condition to this one by a specialization proof. A little idea shows that the proposition 2.1 is suffices to prove the theorem 1.2.

The results of (Flynn et al., 1995) may be viewed as some positive argument nevertheless, particularly from this we do not have many other examples, (however, see Theorem 3.1). If true, this would reveal the uniform boundedness of torsion of abelian differences and other analogous guess. Breiding et al. (2018) for a more detailed of algebraic varieties.

In general, the set of periodic points is of course not finite. However, one can often use some geometric proofs to deduce finiteness of the number of periodic points from Theorem 1.2.

\section{Preliminary}

\section{Proposition 2.1}

Let $R$ be a discrete valuation ring with quotient field $K$ and residue field $K$. Assume $\mathcal{X}$ be a proper scheme of finite type over $\operatorname{Spec}(R)$ and $f: \mathcal{X} \rightarrow \mathcal{X}$ an $R$-morphism. Assume that the results of the theorem hold for $f$ confined to the special fiber and that for each $\varepsilon>-\frac{1}{2}$ there are only finitely many roots of unity covered in all extensions of $k$ of degree 
$\leq 1+2 \varepsilon$. Then the same holds for $f$ restricted to the generic fiber, except possibly in the condition characteristic of $K$ is zero and charactrestic of $K$ is $2+\varepsilon>0$, when the result holds modulo powers of $2+\varepsilon$.

\section{Proof}

Let $\varepsilon=-1$ if ccharacteristic of $K$ is equal zero. The assumption imply that by substituting $f$ with a suitable power we may assume that all the $f$-periodic points in $\mathcal{X}(k)$ have period a power of $2+\varepsilon$. Let $P$ be a $f$-periodic point in $\mathcal{X}(K)$. By substituting $f$ by $f^{(2+\varepsilon)^{1+2 \varepsilon}}$, for some $1+2 \varepsilon$ which may depend on $P$, we may assume that the specialization of $P$ in $\mathcal{X}(k)$ (which exists since $\mathcal{X}$ is proper) is a constant point of $f$ restricted to the special fiber. Let $Z$ be the Zariski closure of the $f$-orbit of $P . Z$ is finite over $\operatorname{Spec}(R)$ with a unique closed point, therefore is equal to $\operatorname{Spec}(A)$ where $A$ is a finite, local $R$-algebra (with rank equal to the period of $P$ ) which, since $A$ is reduced, is torsion free as an $R$-module.

The main viewing of the argument is that $f$ registered to $Z$ covers an automorphism of finite order of $A$ (which we also symbolize by $f$ ): Since $f$ preserves the orbit of $P$ and $Z$ is reduced, it follows that $f$ induces a map from $Z$ to itself, hence an endomorphism of $A$. $f^{1+2 \varepsilon}$ is the identity on the orbit of $P$ for some $\varepsilon>-\frac{1}{2}$, hence $f^{1+2 \varepsilon}$ is the identity on $A \otimes_{R} K$. Since $A$ is torsion free, it follows that $f^{1+2 \varepsilon}$ is the identity on $A$ furthermore.

Let $m$ be the maximal ideal of $A$. Since $Z$ is a closed subschema of $\mathcal{X}$, it follows that the dimension of $\mathrm{m} / \mathrm{m}^{2}$ is bounded independently of $P$. By the assumption on roots of unity, we may change $f$ by some power, independently of $P$, so that the endomorphism of $\mathrm{m} / \mathrm{m}^{2}$ induced by $f$ is the identity. So, $f$ is a unipotent map with respect to the (exhaustive) refinement of $A$ induced by powers of $m$. This means that the order of $f$, therefore, the period of $P$, is a power of $2+\varepsilon$.

\section{Remarks 2.2}

(i) For any explicitly given example, the proof furnishes an effective method for computing a bound for the periods

(ii) In the non-prop condition one can prove the following result by the methods of this study: Let $S$ be a flat, separated, integral scheme of finite type over $Z$, let $Z$ be a separated scheme of finite type over $S$ and let $f: \mathcal{X} \rightarrow \mathcal{X}$ be an $S$-morphism. If one explains the idea of $f$-periodic points and periods for elements of $\mathcal{X}(S)$ in the clear way, then the set of periods is again bounded (i) (iii)One may also ask whether Theorem 1.2 itself holds without the hypothesis of properness, for example when $\mathcal{X}$ is arbitrary but $f$ is finite.

\section{Lemma 2.3}

Let $X$ be a proper variety over a finitely generated field $K$ of characteristic zero and $f: X \rightarrow X$ a morphism. Suppose that there does not exist any positive dimensional subvariety $Y$ of $X$ such that $f$ gets an automorphism of finite order of $Y$. Then the number of $f$-periodic points in $X(K)$ is finite.

\section{Proof}

Theorem 1.2 implies that $f$ gets an automorphism of finite order on the closure of the set of $f$-periodic points in $X(K)$.

The following gives a valid method for examine the assumption of the previous lemma.

\section{Lemma 2.4}

Let $X$ be a projective variety over a field $K$ and $f: X \rightarrow X$ a morphism. Suppose there exists a line bundle $L$ on $X$ such that $f^{*}(L) \otimes L^{-1}$ is ample. Then there is no positive dimensional subvariety $Y$ of $X$ such that $f$ gets an automorphism of finite order of $Y$.

\section{Proof}

By substituting $X$ by $f^{1+2 \varepsilon}(X)$ for some large $1+2 \varepsilon$, we may assume that $f$ is a finite morphism. Suppose there occurs a $Y$ as above and assume that $f^{m} \mid Y$ is the identity of $Y$. Then:

$$
f^{m}(L) \otimes L^{-1}=\bigotimes_{i=1}^{m-1} f^{i^{*}}\left(f^{*}(L) \otimes L^{-1}\right)
$$

By claim $f^{*}(L) \otimes L^{-1}$ is ample so $f^{m}(L) \otimes L^{-1}$, being a tensor product of ample bundles, is also ample. $f^{m}(L) \otimes L^{-1} \mid Y$ is trivial, so it follows that $Y$ must be 0-dimensional.

In this status, is also ample finiteness can also be proved using heights, see for example (Kawaguchi, 1999). One usefulness of our method is that it applies also when $f$ is an automorphism, in which condition an ample $L$ as above cannot exist.

Using this, one can extend the finiteness results of (Silverman, 1991) to apply to all automorphisms $f$ of projective algebraic varieties $X$ for which 1 is not an eigenvalue of $f$ on $N S(X)_{Q}$.

Proposition 2.1 clarifies that one also has boundedness of periods for $2+\varepsilon$-acid fields, up to powers of $2+\varepsilon$, as long as the variety and the morphism extend to the ring of integers. We now explain that in fact we can bound the extra powers of $2+\varepsilon$. 


\section{Theorem 2.5}

Let $\mathcal{O}$ be the ring of integers in $K$, a finite extension of $Q_{2+\varepsilon}$ and let $\mathcal{X}$ be a proper scheme of finite type over $\operatorname{Spec}(\mathcal{O})$. Then there exist a constant $M>0$ such that for any $\mathcal{O}$-morphism $f: \mathcal{X} \rightarrow \mathcal{X}$, the periods of the $f$-periodic points of $\mathcal{X}(K)$ are all less than $M$.

If $X$ is any variety over a finite field $k$ then it is clear that a proof similar to the theorem holds for $X(k)$ : Since this is a finite set the periods are bounded above by $|X(k)|$ and therefore are bounded independently of the morphism. To bound the powers of $2+\varepsilon$ that happen, one views from the proof of Proposition 2.1 that it is sufficient to prove the following.

\section{Proposition 2.6}

Let $\mathcal{O}$ be the ring of integers in $K$, a discrete valuation ring of characteristic zero with residue field $k$ of characteristic $p$. Let $(A, m)$ be a local sub-O-Olgebra of $\mathcal{O}^{p^{n}}$ of rank $p^{n}$ which is kept by the automorphism $\sigma$ given by cyclic permutation of the coordinates. So, assume that acts trivially on $m / m^{2}$. Consequently, $n<r=v(p)$ if $p>2$ and $n \leq r$ if $p=2$, where $v$ is the normalized valuation on $K$.

\section{Proof}

Suppose that $n \geq r$ if $p>2$ and $n>r$ if $p=2$. Since oacts trivially on $\mathrm{m} / \mathrm{m}^{2}$, it follows that $\sigma^{p^{t}}$ acts trivially on $\mathrm{m} / \mathrm{m}^{t+2}$ for all $t \geq 0$. So , by substituting $A$ by a quotient algebra corresponding to the Zariski closure in $\operatorname{Spec}(A)$ of the $\sigma^{p^{n-1}}$ orbit of any $\mathcal{O}$ valued point, we get a local rank $p$ subalgebra of $\mathcal{O}^{p}$ which is stable under (the new) $\sigma$ and such that $\sigma$ acts trivially on $m / m^{r+1}\left(m / m^{r+2}\right.$ if $\left.p=2\right)$.

For $a$ in $A$ we denote by $v(a)$ the minimum of the valuations of the coordinates.

Let:

$$
U(m)=\left\{a \in m \mid v\left(a_{i}\right) \neq v\left(a_{j}\right) \text { for some } i, j\right\}
$$

and let:

$$
P(m)=\{a \in U(m) \mid v(a) \leq v(b), \forall b \in U(m)\} .
$$

Suppose $v(a)=1$ for $a \in P(m)$. Since $P(m) \subset U(m)$, it follows that $v\left(\sigma^{2}(a)-a\right)=1$ for some $s$, which in turn implies that $\sigma^{2}(a)-a \notin m^{2}$.

This is a contradiction, therefore, $(a)>1, \forall a \in P(m)$. Also, one easily sees that any element of $m$ can be written as $a=x+b$ with $x \in \pi \cdot \mathcal{O}$ and $x \in U(m) \cup\{0\}$, where $\pi$ in $\mathcal{O}$ is a uniformizing parameter.

Now let $a \in P(m)$ and consider $\sigma(a)-a$.
Letting $t=r+1$ if $p>2$ and $t=r+2$ if $p=2$, we view that:

$$
\sigma(a)-a=\sum_{i} \prod_{j=1}^{t}\left(x_{i, j}+b_{i, j}\right)
$$

with $x_{i, j} \in \pi \cdot \mathcal{O}$ and $b_{i, j} \in U(m) \cup\{0\}$. Expanding the results and using the fact that $v\left(b_{i, j}\right)>1$, we see that:

$$
\sigma(a)-a=\sum_{k} z_{k} d_{k} \bmod \pi^{v(a)+t}
$$

with $x, z_{k} \in \pi^{t-1} \cdot \mathcal{O}$ and $d_{k} \in P(m)$. Moreover, using the fact that $a$ is in $U(m)$, one sees that $v(x)=v(\sigma(a)-a)=v(a)$. Thus, we get:

$$
\begin{array}{r}
0=\sigma^{p}(a)-a=\sum_{i=0}^{p=1} \sigma^{i}(\sigma(a)-a) \\
=p \cdot x+\sum_{k}\left(\sum_{i=0}^{p-1} \sigma^{i}\left(d_{k}\right)\right) \bmod \pi^{v(a)+t} .
\end{array}
$$

Now the $d_{k}$ 's are also in $P(m)$, so we have:

$$
\sigma\left(d_{k}\right)-d_{k}=w_{k} \bmod \pi^{v(a)+t-1}
$$

With $w_{k}$ in $\pi^{t} \cdot \mathcal{O}$. This implies that:

$$
\sum_{i=0}^{p-1} \sigma^{i}\left(d_{k}\right)=p \cdot d_{k}+\frac{(p-1) p}{2} w_{k} \bmod \pi^{v(a)+t-1} .
$$

Replacing this in Eq. (1) (using that the $z_{k}{ }^{\prime} s$ are in $\pi \cdot \mathcal{O}$ ) we obtain:

$$
p \cdot x+\sum_{k} z_{k}\left(p \cdot d_{k}+\frac{(p-1) p}{2} w_{k}\right)=0 \bmod \pi^{v(a)+t}
$$

We have:

$$
v(x)=v(a)=v\left(d_{k}\right)=v\left(w_{k}\right), v\left(z_{k}\right) \geq t-1
$$

and $v(p)=r$. By the choice of $t$ it follows that the only term in the above equation with valuation less than or equal to $v(a)+r$ is $p \cdot x$. This is a contradiction since $t>r$.

\section{Remark 2.7}

The hypothesis of properness is used only to guarantee the existence of specializations. If we consider an arbitrary separated scheme $\mathcal{X}$ of finite type over $\operatorname{Spec}(\mathcal{O})$, consequently, we get boundedness of the periods for the set of periodic points in $\mathcal{X}(\mathcal{O})$. 
Theorem 2.5 can be provided to the case of some birational maps; this can be used to extend to the $2+\varepsilon$ acid case some of the results of $S$. Marcello (2000) on automorphisms of affine spaces. For $X$ a variety over a field $K$ and $f: X \rightarrow X$ a rational map, we will say that $P \in X(K)$ is $f$-periodic if $f$ is defined at $P, f(P), f^{2}(P), \ldots$ and $f^{1+2 \varepsilon}(P)=P$ for some $\varepsilon>-\frac{1}{2}$.

\section{Main Results}

\section{Theorem 3.1}

Let $\mathcal{O}$ be the ring of integers in $K$, a finite extension of $\mathcal{Q}^{2+\varepsilon}$ and let $\mathcal{X}$ be a proper integral scheme of finite type over $\operatorname{Spec}(\mathcal{O})$. Consequently, there exist a constant $M>0$ such that for any birational map $f: X \rightarrow X$ with $\mathrm{Z}\left(f^{1+2 \varepsilon}\right) \cap \mathrm{Z}\left(f^{-1-2 \varepsilon}\right)=\varnothing$ for all $\varepsilon>-\frac{1}{2}$ the periods of the $f$ periodic points of $\mathcal{X}(K)$ are all less than $M$. Here for any $m \in Z, Z\left(f^{m}\right)$ denotes the exceptional locus of $f^{m}$.

\section{Proof}

The proof is basically the same as that of Theorem 2.5: Let $Z$ be the Zariski closure of the orbit of a periodic point in $P \in \mathcal{X}(K)$. We cannot conclude as before that $f$ induces an automorphism of $Z$, but the assumptions imply that if $Z_{1}$ is a joined component of $Z$, then $\forall 1+2 \varepsilon$ such that $f$ ${ }^{1+2 \varepsilon}\left(Z_{1}(K)\right) \cap Z_{1}(K) \neq \varnothing, f^{1+2 \varepsilon}$ does induce an automorphism of $Z_{1}$. Since the residue field of $\mathcal{O}$ is finite, the number of connected components of $Z$ is bounded independently of $f$. This allows us to apply Proposition 2.6 and the method of Proposition 2.1 to conclude the proof.

\section{Corollary 3.2}

Let $\mathcal{O}$ be the ring of integers in $K$, a discrete valuation ring of characteristic zero with residue field $k$ of characteristic $(2+\varepsilon)$. Let $(A, m)$ be a local sub- $\mathcal{O}$-algebra $\mathcal{O}^{(2+\varepsilon)^{(1+2 \varepsilon)}}$ of rank $(2+\varepsilon)^{(1+2 \varepsilon)}$ which is preserved by the automorphism $\sigma$ given by cyclic permutation of the coordinates. Hence, suppose that $\sigma$ acts trivially on $\mathrm{m} / \mathrm{m}^{2}$. Then $(1+2 \varepsilon)=v^{2}(2+\varepsilon)$ if $\varepsilon>0$, where $v^{2}$ is the normalized valuation on $K$.

\section{Proof}

Suppose that $\varepsilon>0$. Since $\sigma$ acts trivially on $\mathrm{m} / \mathrm{m}^{2}$, it follows that $\sigma^{(2+\varepsilon)^{(1+\varepsilon)}}$ acts trivially on $m / m^{(3+\varepsilon)} \forall, \varepsilon>-1$. Thus, by changing $A$ by a quotient algebra corresponding to the Zariski closure in $\operatorname{Spec}(A)$ of the $\sigma^{(2+\varepsilon)^{(1 \varepsilon)}}$ orbit of any $\mathcal{O}$ valued point, we get a local rank $(2+\varepsilon)$ subalgebra of $\mathcal{O}^{(2+\varepsilon)}$ which is stable under $\sigma$ and such that $\sigma$ acts trivially on $m / m^{(2+\varepsilon)}\left(m / m^{(3+\varepsilon)}\right.$ if $\varepsilon>0$.

For $a$ in $A$ we denote by $v^{2}(a)$ the minimum of the valuations of the coordinates. Let $U^{2}(m)=\left\{\mathrm{a} \in m \mid v^{2}\left(a_{i}\right) \neq\right.$ $v^{2}\left(a_{j}\right)$ for some $\left.i, j\right\}$ and let $(m)=\left\{a \in U^{2}(m) \mid v^{2}(a) \leq v^{2}(b)\right.$, $\left.\forall b \in U^{2}(m)\right\}$.

Assume $v^{2}(a)=1$ for $a \in P(m)$. Since $P(m) \subset U^{2}(m)$, it follows that $v^{2}\left(\sigma^{2}(a)-a\right)=1$ for some $s$, which in turn implies that $\sigma^{s}(a)-a \notin m^{2}$.

This is a contradiction, therefore, $v^{2}(a)>1 \forall a \in P(m)$. Also, one easily observes that any element of $m$ can be written as $a=x^{2}+b$ with $x^{2} \in \pi \cdot \mathcal{O}$ and $b \in U^{2}(m) \cup\{0\}$, where $\pi$ in $\mathcal{O}$ is a uniformizing parameter.

Now let $a \in P(m)$ and consider $\sigma(a)$-a. Letting $\varepsilon \geq 0$, we see that:

$$
\sigma(a)-a=\sum_{i} \prod_{i=1}^{l+\varepsilon}\left(x_{i, j}^{2}+b_{i, j}\right)
$$

with $x_{i, j}^{2} \in \pi . \mathcal{O}$ and $b_{i, j} \in U^{2}(m) \cup\{0\}$. Expanding the results and using the fact that $v^{2}\left(b_{i, j}\right)>1$, we see that:

$$
\sigma(a)-a=x^{2}+\sum_{k} Z_{k} d_{k} \bmod \pi^{v^{2}(a)+(l+\varepsilon)},
$$

with $x^{2}, Z_{k} \in \pi^{(l+\varepsilon-1)}$. $\mathcal{O}$ and $d_{k} \in P(m)$. Moreover, using the fact that $a$ is in $U^{2}(m)$, one views that $v^{2}\left(x^{2}\right)=v^{2}(\sigma(a)-a)$ $=v^{2}(a)$. Thus, we obtain:

$$
\begin{aligned}
& 0=\sigma^{(2+\varepsilon)}(a)-a=\sum_{i=0}^{(1+\varepsilon)}\left(\sigma^{i}(a)-a\right) \\
& =(2+\varepsilon) \cdot x^{2}+\sum_{k} Z_{k}\left(\sum_{i=0}^{(1+\varepsilon)} \sigma^{i}\left(d_{k}\right)\right) \bmod \pi^{v^{2}(a)+(l+\varepsilon)} .
\end{aligned}
$$

Now the $d_{k}$ 's are also in $P(m)$, so we have:

$$
\sigma\left(d_{k}\right)-d_{k}=w_{k} \bmod \pi^{v^{2}(a)+(l+\varepsilon)}-1,
$$

with $w_{k}$ in $\pi^{(l+\varepsilon)} . \mathcal{O}$. This implies that:

$$
\sum_{i=0}^{1+\varepsilon} \sigma^{i}\left(d_{k}\right)=(2+\varepsilon) \cdot d_{k}+\frac{(1+\varepsilon)(2+\varepsilon)}{2} w_{k} \bmod \pi^{v^{2}(a)+(l+\varepsilon-1)}
$$

By changing this in Eq. (2) (using that the $d_{k}$ 's are in $\pi \cdot \mathcal{O})$ we find:

$$
(2+\varepsilon) x^{2}+\sum_{k} Z_{k}\left((2+\varepsilon) \cdot d_{k}+\frac{(1+\varepsilon)(2+\varepsilon)}{2} w_{k}\right)=0 \bmod \pi^{v^{2}(a)+(l+\varepsilon)} \text {. }
$$

We have: 


$$
v^{2}(x)=v^{2}(a)=v^{2}\left(d_{k}\right)=v^{2}\left(w_{k}\right), v^{2}\left(Z_{k}\right) \geq l+\varepsilon-1
$$

and:

$$
v^{2}(2+\varepsilon)=1+\varepsilon
$$

By the choice of $l+1$ it follows that the only term in the above equation with valuation less than or equal to $v^{2}(a)+(1+\varepsilon)$ is $(2+\varepsilon) \cdot x^{2}$. This is a contradiction since $l>1+\varepsilon$ and $\varepsilon>0 \Rightarrow l>1$.

\section{Author's Contributions}

All authors equally contributed in this study.

\section{Ethics}

This article is original and contains unpublished material. The corresponding author confirms that all of the other authors have read and approved the manuscript and no ethical issues involved.

\section{References}

Batoréo, M. (2018). On periodic points of symplectomorphisms on surfaces. Pacific Journal of Mathematics, 294(1), 19-40.

doi.org/10.2140/pjm.2018.294.19

Breiding, P., Kališnik, S., Sturmfels, B., \& Weinstein, M. (2018). Learning algebraic varieties from samples. Revista Matemática Complutense, 31(3), 545-593. doi.org/10.1007/s13163-018-0273-6
Fakhruddin, N. (2001, May). Boundedness results for periodic points on algebraic varieties. In Proceedings of the Indian Academy of Sciences-Mathematical sSciences (Vol. 111, No. 2, pp. 173-178). Springer India. doi.org/10.1007/BF02829589

Flynn, E. V., Poonen, B., \& Schaefer, E. F. (1995). Cycles of quadratic polynomials and rational points on a genus-two curve. arXiv preprint math/9508211.

Kawaguchi, S. (1999). Some remarks on rational periodic points. Mathematical Research Letters, 6(5), 495-509. doi.org/10.4310/MRL.1999.v6.n5.a3

Marcello, S. (2000). Sur les propriétés arithmétiques des itérés d'automorphismes réguliers. Comptes Rendus de l'Académie des Sciences-Series I-Mathematics, 331(1), 11-16. doi.org/10.1016/S0764-4442(00)00325-6

Morton, P., \& Silverman, J. H. (1994). Rational periodic points of rational functions. International Mathematics Resesarch Notices, 1994(2), 97-110. doi.org/10.1155/S1073792894000127

Northcott, D. G. (1950). Periodic points on an algebraic variety. Annals of Mathematics, 167-177. doi.org/10.2307/1969504

Pravec, V. (2019). Remarks on definitions of periodic points for nonautonomous dynamical system. Journal of Difference Equations and Applications, 25(9-10), 1372-1381. doi.org/10.1080/10236198.2019.1641496

Silverman, J. H. (1991). Rational points on K3 surfaces: A new canonical height. Inventiones Mathematicae, 105(1), 347-373. doi.org/10.1007/BF01232270 